\title{
Is the Chiral Phase Transition Induced by a Metal-Insulator Transition?
}

\author{
Antonio M. García-García \\ Physics Department, Princeton University, Princeton, New Jersey 08544, USA and \\ The Abdus Salam International Centre for Theoretical Physics, P.O.B. 586, 34100 Trieste, Italy \\ and James C. Osborn \\ Physics Department \& Center for Computational Science, Boston University, Boston, MA 02215, USA \\ (Received on 11 September, 2006)
}

\begin{abstract}
We investigate the QCD Dirac operator with gauge configurations given by a liquid of instantons in the region of temperatures about the chiral phase transition. Both the quenched and unquenched cases are examined in detail. We present evidence of a localization transition in the low lying modes of the Dirac operator around the same temperature as the chiral phase transition. Thus both level statistics and eigenvectors of the QCD Dirac operator at the chiral phase transition have similar properties than those of a disordered conductor at the metal-insulator transition. This strongly suggests the phenomenon of Anderson localization (localization by destructive quantum interference) is the leading physical mechanism in the restoration of the chiral symmetry. Finally we argue that our findings are not in principle restricted to the ILM approximation and may also be found in lattice simulations.
\end{abstract}

Keywords: Localization; Chiral phase transition; Anderson transition; Metal-insulator transition; Level statistics

The spontaneous breaking of the approximate chiral symmetry $(\mathrm{S} \chi \mathrm{SB})$ and its eventual restoration at finite temperature is one of the most important features of the strong interactions. The order parameter associated to this symmetry breaking is the chiral condensate, $\langle\bar{\psi} \psi\rangle$. By definition, the condensate is nothing but a quark loop in momentum space. Thus it should naively vanish as the quark mass goes to zero. In nature the lightest quarks are not massless so a nonzero condensate is expected even in a free theory. However the small quark bare mass can only account for a very small percentage of the chiral condensate, the rest has its origin in the strong non perturbative color interactions of QCD. QCD models whose gauge configurations are given by an interacting liquid of instantons [1] provide an adequate theoretical framework to understand $\mathrm{S} \chi \mathrm{SB}$. Thus, based on the semiclassical picture of a QCD vacuum dominated by instantons, it has been suggested [2] that the $\mathrm{S} \chi \mathrm{SB}$ in QCD and the phenomenon of conductivity may have similar physical origins. Conductivity in a disordered sample is produced by electrons that although initially bound to an impurity may become delocalized by orbital overlapping with nearby impurities. Similarly, in the QCD vacuum, the zero modes of the Dirac operator though initially bound to an instanton may get delocalized due to the strong overlap with other instantons. As a consequence, the chiral condensate becomes nonzero and chiral symmetry is spontaneously broken. In this paper we provide evidence that these analogies can be extended to describe the chiral phase transition in QCD at finite temperature. Specifically, in the context of an Instanton Liquid Model (ILM), we show that around the temperature that the chiral phase transition occurs the low lying eigenmodes of the QCD Dirac operator undergo an Anderson transition (AT), namely, a localization-delocalization transition, characterized by multifractal eigenstates and critical statistics [4].

For the sake of clarity we provide a brief summary of the properties of a disordered system at an AT. We recall that in three and higher dimensions there exists a mobility edge separating localized from delocalized states. In the delocalized region, eigenfunctions are extended through the sample and level statistics are described by random matrix theory (RMT). In the opposite limit, eigenfunctions are exponentially localized and the spectral correlations are described by Poisson statistics. Around the mobility edge eigenstates are multifractal [6] and level statistics are described by critical statistics [8]. Typical features of critical statistics include scale invariant spectrum [11], level repulsion and asymptotically linear number variance.

Thus the level spacing distribution, $P(s)$ (the probability of having two eigenvalues at a distance $s$ ) goes to zero as the spacing $s$ separation does, $P(s) \rightarrow 0 s \rightarrow 0$. The number variance $\Sigma^{2}(\ell)=\left\langle\left(N_{\ell}-\left\langle N_{\ell}\right\rangle\right)^{2}\right\rangle \sim \chi \ell$ for $\ell \gg 1\left(N_{\ell}\right.$ is the number of eigenvalues in an interval of length $\ell)$ is asymptotically linear, as for an insulator $(\chi=1)$, but with a slope $\chi<1(\sim 0.27$ for a 3D Anderson model [13]). Below we show that at the chiral restoration temperature both eigenvalues and eigenvectors of the QCD Dirac operator have similar properties.

\section{INSTANTON LIQUID MODEL}

In this section we highlight the main features of the QCD vacuum model based on instantons to be used in this paper.

Instantons [3] are classical solutions of the Yang-Mills equations of motion which minimize the action in Euclidean space. They are believed to be the leading semiclassical contribution to the bosonic part of the QCD path integral. However the construction of a consistent QCD vacuum based on instantons faces serious technical difficulties. Exact multiinstanton solutions are hard to obtain since the Yang-Mills equations of motion for QCD are nonlinear and therefore a superposition of single instanton contributions is not itself a solution. Additionally quantum corrections may spoil the semiclassical picture implicitly assumed of a QCD vacuum composed of instantons well separated and weakly interacting. These problems have been overcome either by invoking variational principles [5] or by phenomenologically fixing 
certain parameters of the instanton ensemble. The latter case, usually referred to as the instanton liquid model (ILM) [1] (for a modern review see [15]), yields accurate estimates of vacuum condensates and hadronic correlation functions [10] for most light hadrons just by setting the mean distance between instantons to be $\bar{R} \approx 1 \mathrm{fm}$ (which corresponds to a density $N / V \sim 1 \mathrm{fm}^{-4}$ ) and the mean instanton size $\bar{\rho} \approx 1 / 3 \mathrm{fm}$. Lattice simulations have also supported the picture of a QCD vacuum dominated by instantons [14].

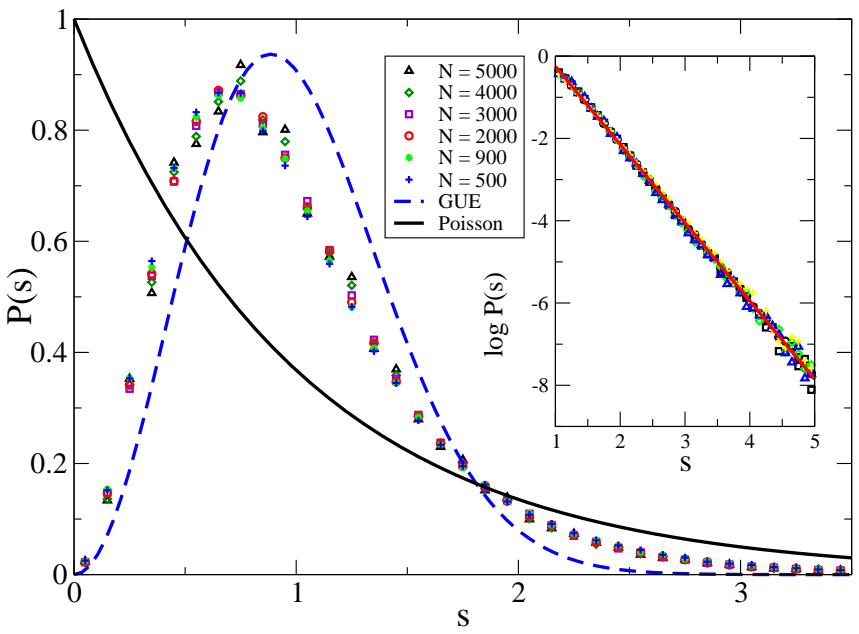

FIG. 1: Level spacing distribution $P(s)$ in the bulk for the quenched ILM at $T=200 \mathrm{MeV}$ for different volumes. The inset shows $\log P(s)$ for the tail of the data. The best fit (solid line) corresponds to a slope -1.64 .

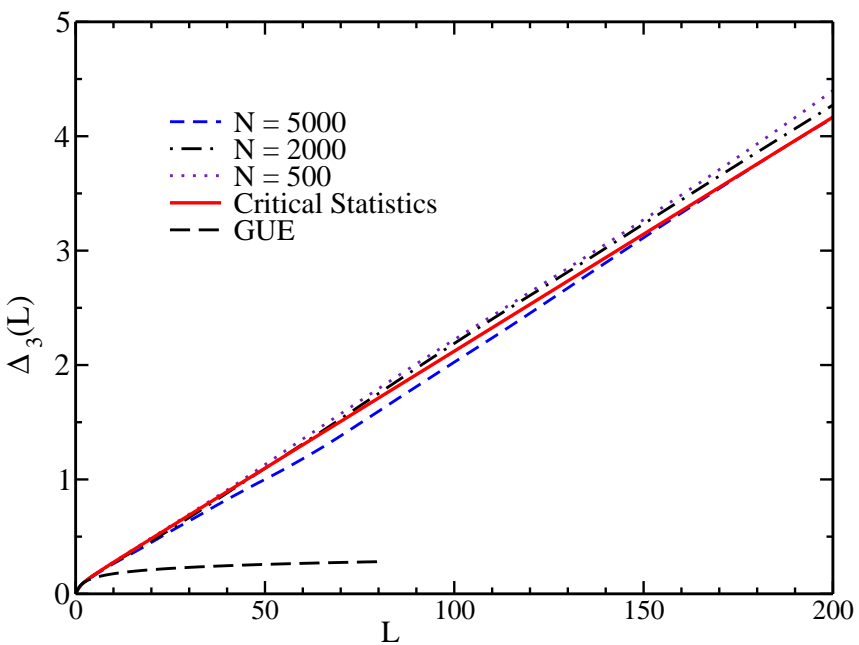

FIG. 2: Spectral rigidity in the bulk for the quenched ILM at $T=200$ $\mathrm{MeV}$ for different volumes. The result has very little size dependence and agrees well with the prediction of critical statistics (solid line).

\section{A. Technical details of the numerical simulation}

The ILM partition function for $N_{f}$ quark flavors with masses $m_{k}$ is given by

$$
Z_{\text {inst }}=\int D \Omega e^{-S_{\mathrm{YM}}} \prod_{k=1}^{N_{f}} \operatorname{det}\left(\not D+m_{k}\right),
$$

where the integral is over the positions, sizes and orientations of the instantons and $S_{\mathrm{YM}}$ is the Yang-Mills action. The fermion determinant is evaluated in the space of the fermionic zero modes of the instantons. For further discussion of this partition function we refer to [15]. We just mention that we use the standard phenomenological value of the instanton density $N / V=1 \mathrm{fm}^{-4}$. For the sake of simplicity we have kept this density fixed as the temperature is increased. We justify this approximation based on the fact that even for temperatures close to the chiral phase transition the instanton density is still sizable (around $0.6 \mathrm{fm}^{-4}[15]$ ). Indeed in previous simulations the drop in the density was ruled out as the physical mechanism leading to the chiral phase transition [15].

All units in the ILM are typically given in terms of the QCD scale parameter $\Lambda$ which we have simply set to 200 $\mathrm{MeV}$. This choice is sufficient for our purposes since we are not concerned with making quantitative predictions about the position of the QCD chiral phase transition, but instead are interested in more general features of the transition that we don't expect to be very sensitive to a fine tuning of the parameters of the ILM. We therefore use the ILM as a qualitative model for QCD at temperatures around the chiral phase transition. We also stress that it provides a reasonable description of $\mathrm{S} \chi \mathrm{SB}$ and many hadronic correlation functions both at zero and at finite temperature [10]. Furthermore the ILM has modest computational requirements and allows us to go to fairly large volumes and get good statistics.

In this paper we present results for both the quenched $N_{f}=0$ and the unquenched $N_{f}=2$ cases $\left(N_{f}=2\right.$ with $m_{u}=$ $\left.m_{d}=0\right)$. The partition function, equation (1), is evaluated using a standard Metropolis algorithm. In the quenched case we performed between 2000 to 10000 measurement sweeps for each set of parameters after allowing around 1000 sweeps for thermalization. Results are presented for ensembles of up to $N=5000$ instanton and anti-instantons. In the unquenched case we were only able to investigate ensembles of up to $N=700$ instantons and anti-instantons. We also discarded the first 1000 sweeps in each simulation and did between 1000 to 5000 sweeps per ensemble. Thermodynamic properties of the model have already been addressed in [15] and will not be discussed here. We will focus mainly on observables such as level statistics and eigenvector scaling properties of the QCD Dirac operator which are especially well suited for studying Anderson localization effects [18]. All the spectral correlators are calculated from the unfolded spectrum. This procedure scales the eigenvalues so that the spectral density on a spectral window comprising several level spacings is unity but it does not remove the small fluctuations about the mean density that provide fundamental information about the system.

Finally we recall that in the ILM the effective disorder pa- 
rameter is the temperature since we have fixed the density of instantons. One of the most challenging tasks of the numerical calculation is to accurately locate the temperature at which the AT occurs. In all cases in the paper this temperature has been estimated by the finite size scaling method introduced in [11] in the context of disordered systems. In essence this method consists of computing a spectral correlator, such as the level spacing distribution $P(s)$ or the number variance $\Sigma^{2}(\ell)$, for different sizes and then finding the temperature at which it becomes size independent.

\section{LOCALIZATION IN THE INSTANTON LIQUID MODEL: THE QUENCHED CASE}

In this section we present results for the quenched ILM at nonzero temperature. Without dynamical fermions there is not technically a chiral phase transition since there is no chiral symmetry. However one can in principle study the "quenched" quark condensate in a purely gluonic background through the Banks-Casher relation [16]. However, in the quenched ILM, the spectral density seems to diverge close to the origin [2] (similarly to recent lattice results with overlap fermions [9]) thus suggesting a likewise divergent "quenched" quark condensate. In view of these facts, the transition to localization (see below) we have observed in the quenched QCD Dirac operator at finite temperature both close to origin and in the bulk of the spectrum can't be linked directly to either of these phenomena. We still consider the quenched ILM results to be of interest as we have found a very clear example of a mobility edge with features strikingly similar to those of a 3D disordered system at the AT.

First we examine the properties of eigenstates in the bulk of the spectrum. This region is not directly related to the chiral transition however it is a very clear example of a mobility edge in the ILM. Moreover since it affects a larger range of eigenmodes we can get better statistics. By using the finite scaling method introduced in [11] we have observed a mobility edge separating localized from delocalized eigenstates in the range $T \sim 150-250 \mathrm{MeV}$. As the temperature decreases the location of the mobility edge moves to the end of the spectrum. For $T<170 \mathrm{MeV}$ the results are less reliable since the mobility edge is located almost in the end of the spectrum where truncation effects are larger. Unlike the unquenched case, where the first temperature to study is in principle dictated by the restoration temperature, we do not have a special reason to choose a specific temperature to investigate the AT.

Following the literature in disordered systems we have investigated the temperature $T \sim 200 \mathrm{MeV}$ such that the mobility edge is located around the center of the spectrum, namely, if the spectrum spans a spectral window $\left[0, e_{f}\right]$, the center corresponds with a region around $\sim e_{f} / 2$.

The level statistics in this spectral region have all the signatures of an AT including a spectrum that is scale invariant to high degree. As observed in figures 1 and 2 both short range statistics such as the level spacing distribution, $P(s)$, and long range correlators such as the spectral rigidity $\Delta_{3}(\ell)=\frac{2}{\ell^{4}} \int_{0}^{\ell}\left(\ell^{3}-2 \ell^{2} x+x^{3}\right) \Sigma^{2}(x) d x$ do not depend on the system size for volumes (number of instantons) ranging from $N=500$ to $N=5000$. Level repulsion is still present as for a metal but the spectral rigidity (see figure 2 ) is asymptotically linear with a slope that corresponds to $\chi \sim 0.29 \pm 0.02$ in fair agreement with the value for a $3 \mathrm{D}$ disordered system at an AT of $\chi \sim 0.27 \pm 0.02$ [13]. Furthermore the exact form of the spectral rigidity follows closely the prediction of critical statistics, equation (31) in [17] with $h$, a free parameter, set to a value of 0.62

We now investigate the multifractality of the eigenstates by looking at the scaling of $P_{q}=\int d^{d} r|\psi(\mathbf{r})|^{2 q} \propto L^{-D_{q}(q-1)}$, where $D_{q}$ is a set of different exponents describing the transition and $L \propto N^{1 / 3}$ is the spatial system size. The second moment $P_{2}$ is usually referred to as the inverse participation ratio (IPR).

In order not to consider eigenstates outside the critical region we have taken only $10 \%$ of the eigenvectors around the center of the spectrum for $T=200 \mathrm{MeV}$. By fitting $P_{2}$ (also known as the inverse participation ratio or IPR) for different volumes, we have obtained a value of $D_{2} \sim 1.5 \pm 0.1$ in agreement with that of a 3D disordered system at the AT [18].

Our spectral and eigenfunction analysis shows that at $T=$ $200 \mathrm{MeV}$ there is mobility edge around the central part of the spectrum in the quenched ILM. We have also observed similar critical features in the region close to the origin at temperatures around 110-140 MeV. Here we expect the appearance of the mobility edge to be related to $\mathrm{S} \chi \mathrm{SB}$, However the analysis is complicated by the accumulation of very small eigenvalues typical of the quenched approximation in QCD. Additionally numerical results are less conclusive due to poor statistics. Despite these limitations we have also observed critical statistics and multifractal eigenstates with $D_{2} \sim 0.8 \pm 0.1$ (more sparse than in the bulk).

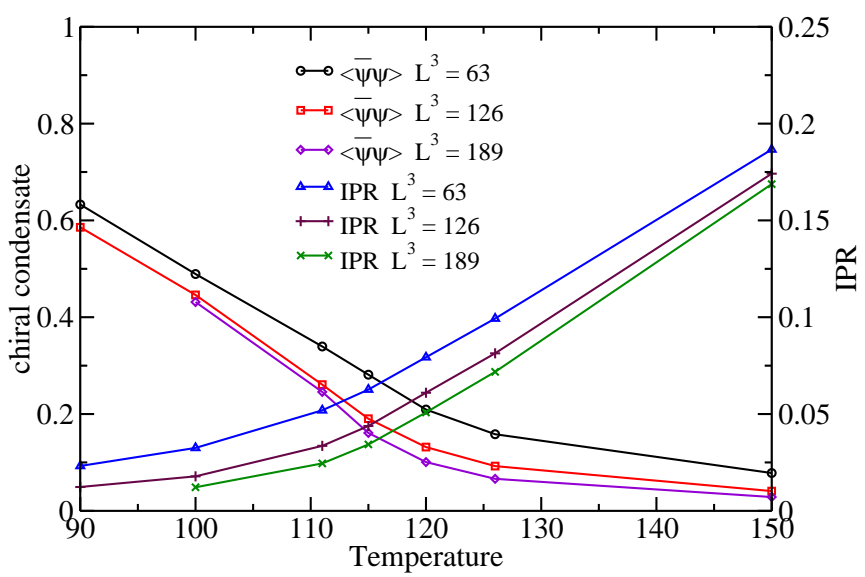

FIG. 3: Chiral condensate and inverse participation ratio (IPR) of the lowest eigenmode for the ILM with two massless quarks. 


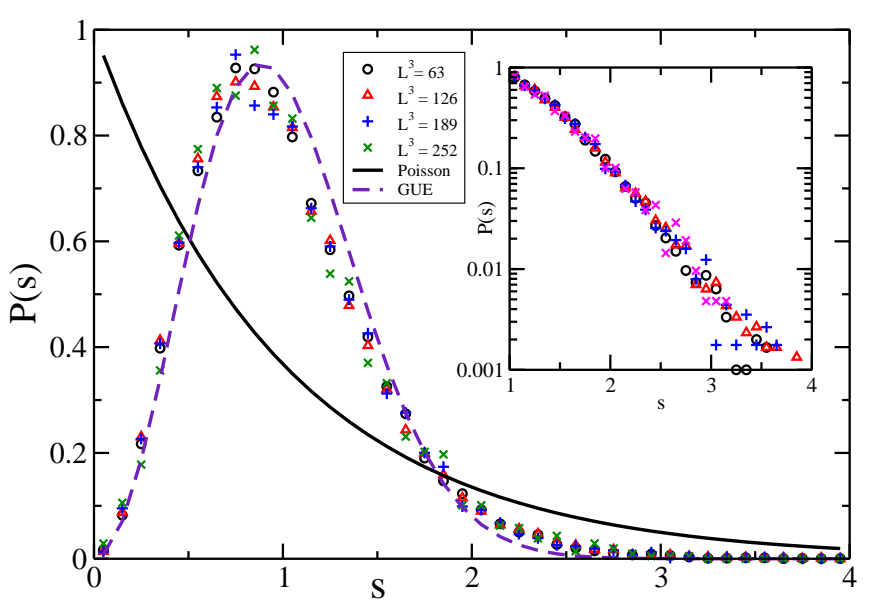

FIG. 4: Level spacing distribution $P(s)$ at the origin for the unquenched ILM at $T=115 \mathrm{MeV}$ for different system sizes.

\section{CHIRAL RESTORATION AND ANDERSON TRANSITION: THE UNQUENCHED CASE}

We now study eigenvalues and eigenvectors of the QCD Dirac operator in an ensemble with two massless quark flavors. In this case the chiral condensate associated with $\mathrm{S} \chi \mathrm{SB}$ is finite. Around the critical temperature, the condensate approaches zero signaling the chiral phase transition. Since we have two massless flavors we expect to find a second order chiral phase transition.

We focus our attention on the spectral region close to origin since our aim is to relate the chiral phase transition with a transition to localization (AT) of the QCD Dirac operator eigenmodes. By looking at the chiral condensate versus temperature for a range of system sizes (figure 3) we see that the condensate approaches zero around temperatures of $115-120$ $\mathrm{MeV}$ in a manner consistent with a second order phase transition. At the same time the condensate is falling we see the IPR of the lowest eigenmode begin to rise signaling a transition to localization. This finding strongly suggests that both phenomena are intimately related as was first conjectured in Ref.[7].

Next we examine the nature of the localization transition. First we locate, by using the finite size scaling method [11], the AT for the lowest lying eigenmodes at $T \sim 115-120 \mathrm{MeV}$. At this temperature we see that eigenvalue statistics such as the level spacing distribution (figure 4) are nearly scale invariant, there is level repulsion but the tail of $P(s)$ is exponential as at the AT. We also looked at the scaling of eigenmode moments $P_{q}$ in the lowest eigenmode at $T=120 \mathrm{MeV}$ for system sizes ranging from $L^{3}=63-252$. This yielded a set of fractal dimensions $D_{2}=1.3 \pm 0.2, D_{3}=0.9 \pm 0.2, D_{4}=0.7 \pm 0.3$ and $D_{5}=0.7 \pm 0.3$ Although higher volumes would be desirable to fully confirm the multifractal scaling of the eigenstates, it is encouraging that the IPR of comparatively similar temperature $(T \sim 100 \mathrm{MeV})$ is comparable to the one for a fully metallic sample. We also mention that we observed a mobility edge on the bulk of the spectrum at a slightly higher temperature with properties similar to the quenched case.

At this point a natural question to address is whether the results here reported concerning the Anderson transition in the ILM may also be present in more realistic models of the strong interactions. Although no conclusive answer can be given to this question we would like to point out the reasons why we have found an Anderson transition in the ILM. As is known, unlike the zero temperature case where the decay is powerlaw, the fermionic zero modes in the field of an instanton at nonzero temperature (usually called a caloron) have an exponential tail $e^{-r T}$ in the spatial directions and are oscillatory in the time direction [15]. This suggests that the overlap among different zero modes is essentially restricted to nearest neighbors in the spatial directions. However, in the time direction different zero modes strongly overlap due to the oscillatory character of the eigenmodes. This situation strongly resembles a 4D disordered conductor in the tight binding approximation (only nearest neighbor hopping) with one dimension (time) much smaller than the rest so the system can be considered effectively three dimensional. It is well established that such a system may undergo an AT depending on the disorder strength. In our case the role of disorder is played by temperature since the wavefunction decays as $\sim e^{-r T}$. From this discussion it is natural to find an Anderson transition in the ILM for a particular value of the temperature as first suggested in Ref. [7].

The principal ingredient to reach the AT is thus an exponential decay of the eigenmodes explicitly depending on the temperature together with the possibility to tune the effective range of the exponential in order to reach the transition region. Any theory with these features very likely will undergo an AT for some value of the parameters (see Ref. [12] for a more detailed discussion). It is therefore quite possible that the QCD vacuum has similar properties. Even if the objects responsible for localization are not the classical instantons this scenario could still be realized if the interactions behave in a similar way. We refer to [12] for a more detailed account of this interesting issue.

In summary we have found that in the unquenched ILM the chiral restoration transition occurs around the same temperature as the localization transition close to the origin. This indicates that both phenomena are closely related. From a physical point of view this is not surprising since the appearance of the condensate is deeply linked [16] with the delocalization of the zero modes in the instanton vacuum due to long range instanton induced interactions. It will be very interesting to repeat these same studies in lattice simulations where the chiral transition also coincides with a deconfinement transition and additionally to see if the detailed structure of the topological objects affects the nature of the localization transition. 
Phys. Lett. B 147, 351 (1984); hep-ph/9602375; J.C. Osborn and J.J.M. Verbaarschot, Phys. Rev. Lett. 81, 268 (1998); Nucl. Phys. B 525, 738 (1998); R.A. Janik, M.A. Nowak, G. Papp, and I. Zahed, Phys. Rev. Lett. 81, 264 (1998).

[3] A. Belavin, A. Polyakov, A. Schwartz, and Y. Tyupkin, Phys. Lett. 59, 85 (1975); G. 't Hooft, Phys. Rev. Lett. 37, 8 (1976).

[4] P.W. Anderson, Phys. Rev. 109, 1492 (1958)

[5] D. Diakonov and V. Petrov, Nucl. Phys. B 245, 259 (1984).

[6] H. Aoki, J. Phys. C 16, L205 (1983); F. Wegner, Z. Phys. B 36, 209 (1980).

[7] D. Diakonov, Lectures at the Enrico Fermi School in Physics, Varenna, hep-ph/9602375.

[8] V.E. Kravtsov and K.A. Muttalib, Phys. Rev. Lett. 79, 1913 (1997).

[9] J. Kiskis and R. Narayanan, Phys.Rev. D 64, 117502 (2001).

[10] T. Shafer, E. Shuryak, and J.J.M. Verbaarschot, Nucl. Phys. B
412, 143 (1994).

[11] B.I. Shklovskii, et.al., Phys. Rev. B 47, 11487 (1993).

[12] Antonio M. Garcia-Garcia, James C. Osborn, Nucl. Phys. A 770, 141 (2006).

[13] D. Braun, G. Montambaux, and M. Pascaud, Phys. Rev. Lett. 81, 1062 (1998).

[14] M.-C. Chu, et. al., Phys. Rev. D 49, 6039 (1994); C. Michael and P.S. Spencer, Phys. Rev. D 52 (1995) 4691.

[15] T. Schäfer and E. Shuryak, Rev. Mod. Phys. 70, 323 (1998).

[16] T. Banks and A. Casher, Nucl. Phys. B 169, 103 (1980).

[17] A.M. Garcia-Garcia and J.J.M. Verbaarschot, Phys. Rev. E 67, 046104 (2003).

[18] T. Ohtsuki and T. Kawarabayashi, J. Phys. Soc. Jpn. 66, 314 (1997). 